\title{
DIPLOME INTER UNIVERSITAIRE DE SEXOLOGIE
}

La formation à la pratique de la Sexologie est assurée en France, depuis une vingtaine d'années, par des enseignements très divers (universitaires ou non) conduisant à des niveaux de qualification variables et ce d'autant plus que l'accès n'en est pas réservé aux seuls médecins. Ceci aboutit à une situation paradoxale : les médecins ayant acquis une bonne compétence ne peuvent en faire état alors que tous les non-médecins peuvent exercer leur pratique sans aucun contrôle.

S'appuyant sur ce constat, l'Association Inter Hospitalo Universitaire de Sexologie (AIHUS) est intervenue dès 1994 auprès du Conseil National de l'Ordre afin d'entreprendre une réflexion sur les conditions d'une reconnaissance officielle de la pratique de la Sexologie par les médecins et d'un diplôme garantissant leur compétence.

Le Bureau du Conseil National a mis en place un groupe de travail chargé d'étudier ces propositions. Ce groupe composé de représentants de l'AIHUS, de médecins libéraux concernés par la pratique de la sexologie et du Secrétaire de la Conférence des Doyens des Facultés de Médecine, a remis le 29 Juin 1995 un rapport dont nous relevons ci-dessous les conclusions :

Pratique psycho-médicale, nécessitant une approche globale de l'homme, suivie souvent d'une prise en charge pluridisciplinaire, la sexologie s'inscrit dans l'évolution de nos sociétés (éducation sexuelle, santé publique, conseils, thérapie).

Elle nécessite un savoir-faire particulier du médecin et un respect strict des règles du Code de Déontologie.

Un pré-acquis de sexologie devrait être enseigné dans le cursus normal des études médicales et une formation complémentaire par un D.I.U. national est sur le point d'être instaurée.

La Formation Médicale Continue est ici indispensable.

L'exercice de cette discipline est pollué par l'existence d'une pratique non médicalisée, incontrôlée et difficilement contrôlable. Il est souhaitable de la réserver à des médecins formés.

D'où la nécessité d'une formation universitaire homogène sur le territoire national tant initiale que complémentaire (D.I.U., voire capacité). La mention de ce titre, réservée aux médecins formés, devrait être autorisée par le Conseil National de l'Ordre (article 67 du Code de Déontologie) sous couvert du Ministre de la Santé.

Plusieurs universités se sont associées pour élaborer un projet commun de DIU de Sexologie.

Ce projet a été déposé au cours du mois de mai 1996 auprès des instances universitaires compétentes pour décider de son habilitation et des conditions de sa mise en place. L'ensei- 
gnement devrait débuter dès le mois d'octobre 1996 ; pour cette raison nous avons décidé de présenter dès maintenant à nos lecteurs des informations qui devront être confirmées dans les prochaines semaines.

\section{Etablissements délivrant la formation}

Les Universités ou groupe d'universités de Bordeaux, Lyon, Marseille, Montpellier-Nimes, Nantes/Rennes (coordonnant conjointement l'enseignement donné par elles-mêmes et les universités d'Angers, Brest, Caen, Poitiers et Tours), Paris Bobigny, Toulouse.

\section{Organisation à l'échelon national}

Une commission pédagogique nationale est constituée de 3 représentants par Université ou groupe d'Universités.

Cette commission procède à l'adaptation et l'harmonisation du contenu pédagogique $d u$ DIU. Elle désigne en son sein le jury responsable du choix des sujets et de la validation de l'examen national de 3ème année.

\section{Conditions d'inscription}

en 1ère année :

- être titulaire du diplôme de docteur en médecine ou d'un diplôme étranger autorisant l'exercice de la médecine dans le pays d'origine.

- être en 2ème année de DES, ou DIS ou de résidanat en médecine générale.

en 2ème année :

- avoir suivi $80 \%$ des cours de la 1ère année.

- avoir été reçu à l'examen de lère année.

en 3ème année :

- avoir suivi $80 \%$ des cours de la 2ème année

- avoir été reçu à l'examen régional de fin de 2ème année

Le montant annuel des droits d'inscription est fixé par chaque université.

\section{Programme}

\section{CONNAISSANCES FONDAMENTALES BASES DE SEXOLOGIE CLINIQUE}

1ère Année : $60 \mathrm{~h}$

I - BASES SEXOLOGIQUES

\section{1- Biosexologie - 10h}

1-Développement (ontogénèse) des appareils génitaux

2-Anatomie et Physiologie des réaction sexuelles chez l'homme et chez la femme

3-Psycho neuro endocrinologie du désir et du plaisir

4-Comportement sexuel 
2• Développement de la Sexualité et Identité Sexuelle - 10h

1-Développement psychosexuel de l'enfant

2-Construction de la personnalité

3-Identité sexuelle, orientation sexuelle

4-Rôles sexuels

5-Désir, Séduction, Sentiments amoureux

6-Imaginaire et Erotisme

3- Sexualité et Cycles de vie - $8 \mathrm{~h}$

1-Adolescence et sexualité

2-Reproduction et Sexualité

3-Ménopause, Andropause

4-Vieillissement

\section{II - FONDEMENT DE LA SEXUALITE HUMAINE}

1-Histoire de la pensée sexologique

2-Anthropologie et sexualité

3-Phylogénèse et ethologie

4-Sociologie et Sexualité

Normalité Sexuelle

Contrôle Social

5-Lois et Sexualité

6-Religion et Sexualité

7-Art, Esthétique et sexualité

1-La plainte sexologique - La demande et le symptôme sexuel

2-L'anamnèse

3-L'examen clinique. Place du bilan organique

4-Relation médecin-malade

5 -Ethique en sexologie

IV - EXPLORATIONS PARACLINIQUES DE LA PATHOLOGIE SEXUELLE

-Neuro-vasculo-endocrinologie sexuelle

\section{V - ASPECTS ORGANIQUES DE LA PATHOLOGIE SEXUELLE}

$\mathbf{8 h}$

1-Interaction de la pathologie organique avec le comportement sexuel

2-Anomalies organiques et anatomiques

3-Iatrogénicité : retentissement sexuel des traitements médicaux et chirurgicaux et des interventions sur la reproduction chez l'homme et la femme 
2ème Année - $60 \mathrm{~h}$

\section{I - DYSFONCTIONS SEXUELLES MASCULINES}

$8 h$

1-Troubles de l'érection

2-Troubles du désir

3-Troubles de l'éjaculation

4-Dyspareunie

5-Troubles du plaisir

II - DYSFONCTIONS SEXUELLES FEMININES

$8 h$

1-Dyspareunie

2-Vaginisme

3-Troubles du désir

4-Anorgasmie

III - DYSFONCTIONS SEXUELLES DU COUPLE

$4 h$

1-Mariage non consommé

2-Conjugopathies

IV- PSYCHOPATHOLOGIE ET SEXUALITE

$4 h$

1-Retentissement des névroses, des psychopathies et des troubles de la personnalité

2-Paraphilies

V. TRANSSEXUALISME

$\mathbf{2 h}$

VI - MST ET SEXUALITE. SIDA ET SEXUALITE

$\mathbf{2 h}$

VII - HANDICAP ET SEXUALITE

$\mathbf{2 h}$

VII - LES TRAITEMENTS

1-Evaluation dans les différentes approches

(2h)

1-Le patient, le couple, le symptôme

2-Bilan physique

3-Bilan psychologique

2-Analyse des différents systèmes thérapeutiques

-Fondement théorique, Objectifs, Moyens,

-Indications , Contre-indications

3-Présentation des différentes thérapeutiques utilisées

1-Traitement pharmacologique

2-Traitement chirurgical

3-Thérapie sexuelle

-Approche cognito-comportementale 
-Approche psycho-dynamique

-Approche sexo-corporelle

-Approche sexo-analytique

-Approche systémique

-Thérapie de couple

4-Possibilité d'intégration de différentes approches

5-Les thérapies sont différentes des techniques

(Sophrologie, relaxation, hypnose, massage...)

$4 \bullet$ Conduite du traitement

1-Choix pour chaque dysfonction

2-Contrat thérapeutique

3-Suivi thérapeutique

IX - LA PREVENTION : CONSEIL-INFORMATION-EDUCATION

10h

1-Concept de santé sexuelle

2-Education sexuelle :

-Objectifs

-Analyse des programmes

3-Conseil sexologique : Rôle et place des paramédicaux

\title{
ENSEIGNEMENT DIRIGE - APPLICATIONS DES COURS THEORIQUES de 1ère et 2ème Année TRAVAIL DE RECHERCHE EN VUE DU MEMOIRE
}

\author{
3ème année - $60 \mathrm{~h}$
}

\section{0 heures d'Enseignement dirigé}

(Centré sur la pratique en consultation sexologique ou pratique en Education sexuelle, selon option choisie)

L'enseignement théorique est complété par un Enseignement dirigé :

-Présentation de cas vidéo

-Simulations de consultations (jeux de rôle)

-Groupe Balint

-Attitudes et contre attitudes des étudiants

-Supervision thérapeutique

Durée des études : $\quad$-Actuellement sur 3 ans : 180 heures

La Commission Pédagoqiue du Diplôme se réserve la possibilité de faire appel à des experts pour compléter la formation dans des domaines particuliers.

\section{Validation de l'enseignement}

En fin de 1ère année

Examen régional universitaire

Epreuve écrite de 2 heures notée sur 20.

En fin de 2ème année

Examen régional universitaire

Epreuve écrite de 2 heures notée sur 20. 
Ne peuvent accéder à cet examen que les candidats ayant été reçus à l'examen de lère année.

\section{En fin de 3ème année}

Ne peuvent accéder à cet examen que les candidats ayant été reçus à l'examen de 2ème année. Validation nationale comportant une notation sur 20 de :

-1/4 : examen national écrit avec une session en septembre portant sur le programme théorique des 2 premières années,

-1/4 : mémoire personnel de 30 à 40 pages avec bibliographie selon les normes internationales et éventuellement des annexes, soutenu régionalement et validé nationalement.

-1/4 : notes régionales de contrôle continu des lère et 2ème années.

-1/4 : titres et travaux : évaluation par le jury de la formation personnelle.

Les étudiants n'ayant pas obtenu la moyenne lors de la validation nationale, auront la possibilité de redoubler une seule fois.

Chaque année : obligation de présence aux $3 / 4$ des cours, aux ateliers, au Séminaire de Perfectionnement de l'AIHUS ; obligation de remise de commentaires de lectures ; obligation d'assister aux séances de travail sur observations cliniques - note sur 20.

En fin d'études : soutenance d'un mémoire sur un sujet en rapport avec la sexologie clinique, devant un jury qui se réunit une fois par an. - note sur 20. 